\title{
On Factorization of Functional Operators with Reflection on the Real Axis
}

\author{
OLEKSANDR KARELIN, ANNA TARASENKO \\ Institute of Basic Sciences and Engineering \\ Hidalgo State Autonomous University \\ Carretera Pachuca-Tulancingo, km.4.5, \\ Pachuca, Hidalgo, C.P. 42184 \\ MEXICO \\ karelin@uaeh.edu.mx, anataras@uaeh.edu.mx
}

\begin{abstract}
Problems of factorization of matrix functions are closely connected with the solution of matrix Riemann boundary value problems and with the solution of vector singular integral equations. In this article, we study functional operators with orientation-reversing shift reflection on the real axes. We introduce the concept of multiplicative representation of functional operators with shift and its partial indices. Based on the classical notion of matrix factorization, the correctness of the definitions is shown. A theorem on the relationship between factorization of functional operators with reflection and factorization of the corresponding matrix functions is proven.
\end{abstract}

Key-Words: - Factorization, Functional operators, Carleman shift, Reflection, Matrix Riemann boundary value Problem, Partial indices, Operator identities

Received: November 24, 2020. Revised: March 22, 2021. Accepted: April 9, 2021. Published: May 9, 2021.

\section{Introduction}

A large number of works have been devoted to Riemann boundary value problems and to the associated singular integral equations. An important place within this topic is occupied by the study of problems with shift and integral equations with shift. Interest toward these problems has not diminished and remains high.

Since until now the methods to factorize a matrix function in general form are not known, important special cases of function matrices are considered for which effective methods of calculating or estimating partial indices $[1,2]$ and constructing factorization factors $[3,4]$ are proposed.

Some relevant studies can be found in $[5,6]$.

In the article [5], we built operator identities that transform singular integral operators with involutions generated by linear fractional Carleman operators into equivalent vector characteristic operators without shift. This transformation is carried out using invertible operators. The simplicity of shifts allows us to obtain vector singular integral operators without additional operators and to avoid the appearance of compact terms that do not influence the construction of Fredholm theory, but that substantially affect the dimension, kernel structure and methods of finding solutions of the corresponding equations.
So, for a singular integral operator $B$ with involution that changes orientation, the operator identity has the form $H B E=D_{R_{+}}$. Here, $H$ and $E$ are invertible operators and $D_{R_{+}}$is a vector characteristic operator. Based on this approach, applications have been found in which the main method of investigation are operator identities [6-9].

In [10], we proposed new applications of operator identities. A definition of factorization for functional operators with involutive rotation on the unit circle was given, partial indices were defined and their uniqueness was proven.

In this work, operator identities were applied to the study of factorization representations of functional operators with shift-reflection and continuation on the real axis.

In Section 2, we present information about the operator identities considered in our case, where shift is a reflection on the real axis. We also present some auxiliary formulas, which will be used in subsequent sections.

In Section 3, we introduce the definition of factorization for functional operators with reflection and continuation.

In Section 4, we prove the equivalence of factorization for functional operators with reflection and factorization of the corresponding matrix 
functions. Note that this theorem opens up the possibility of transferring well-known results on factorization of matrices of second order to multiplicative representations of functional operators with fractional linear involutive shift.

\section{On operator identities for shift- reflection on the real axis}

Let $\Gamma$ and $\gamma$ be contours, and let $\gamma \subset \Gamma$. The extension of a function $f(t), t \in \gamma$, to $\Gamma / \gamma$ by the value zero will be denoted by $\left(J_{\Gamma \backslash \gamma} f\right)(t), t \in \Gamma$. The restriction of a function $\varphi(t), t \in \Gamma$, to $\gamma$ will be denoted by $\left(C_{\gamma} \varphi\right)(t), t \in \gamma$. Symbol $\left[H_{1}, H_{2}\right]$, will be used for the set of linear bounded operators acting from the Banach space $\left[H_{1}\right]$ on Banach space $\left[H_{2}\right] ;\left[H_{1}\right]=\left[H_{1}, H_{1}\right]$.

Let $R$ denote the real axes, $R_{+}$its positive part and $R_{-}$its negative part. Consider spaces with weight $L_{2}^{2}(R, \rho), L_{2}^{2}\left(R_{+}, \rho\right), \rho(t)=t^{-\frac{1}{4}}$, which are determined in the usual way: $L_{2}^{2}(R, \rho(t))=\left\{f(t): \rho(t) f(t) \in L_{2}(R)\right\}$, $L_{2}^{2}\left(R_{+}, \rho(t)\right)=\left\{f(t): \rho(t) f \rho(t) \in L_{2}\left(R_{+}\right)\right\}$.

We introduce operators on the contour $R$ : let $I_{R}$ be the identity operator and $W_{R}$ be the reflection operator:

$\left(I_{R} \varphi\right)(t)=\varphi(t), \quad\left(W_{R} \varphi\right)(t)=\varphi(-t), \quad t \in R$, $I_{R}, W_{R} \in\left[L_{2}(R)\right], C_{R_{+}} \in\left[L_{2}(R), L_{2}\left(R_{+}\right)\right]$, $J_{R_{-}} \in\left[L_{2}\left(R_{+}\right), L_{2}(R)\right]$.

We write out some relations based on which the operator identities have been obtained [7, 8]. Presently, they will be used for the study of factorization representations of functional operators with Carleman shift-reflection. For this, we need the operators:

$$
\begin{aligned}
M_{R_{+}}\left[\begin{array}{l}
\varphi_{1} \\
\varphi_{2}
\end{array}\right]=\left\{\begin{array}{l}
\varphi_{1}(t), t \in R_{+} \\
\varphi_{2}(t), t \in R_{-}
\end{array}=J_{R_{-}} \varphi_{1}(t)+W_{R} J_{R_{-}} \varphi_{2}(t),\right. \\
M_{R_{+}}^{-1} \varphi=\left[\begin{array}{c}
\varphi(t), t \in R_{+} \\
\varphi(-t), t \in R_{+}
\end{array}\right]=\left[\begin{array}{c}
C_{R_{+}} \varphi(t) \\
C_{R_{+}} W_{R} \varphi(t)
\end{array}\right],
\end{aligned}
$$

$$
\begin{gathered}
Z_{R_{+}}=Z_{R_{+}}^{-1}=\frac{1}{\sqrt{2}}\left[\begin{array}{cc}
1 & 1 \\
1 & -1
\end{array}\right], \\
\left(N_{R_{+}} \zeta\right)(t)=\zeta\left(t^{2}\right), \quad\left(N_{R_{+}}^{-1} \zeta\right)(t)=\zeta\left(t^{\frac{1}{2}}\right), \\
M_{R_{+}}^{-1} \in\left[L_{2}(R), L_{2}^{2}\left(R_{+}\right)\right], M_{R_{+}} \in\left[L_{2}^{2}\left(R_{+}\right), L_{2}(R)\right], \\
N_{R_{+}} \in\left[L_{2}^{2}\left(R_{+}, \rho\right), L_{2}^{2}\left(R_{+}\right)\right], N_{R_{+}}^{-1} \in\left[L_{2}^{2}\left(R_{+}\right), L_{2}^{2}\left(R_{+}, \rho\right)\right] .
\end{gathered}
$$

We define operator $H$ by the composition of the operators

$$
H=N_{R_{+}}^{-1} Z_{R_{+}}^{-1} M_{R_{+}}^{-1}, \quad H \in\left[L_{2}(R), L_{2}^{2}\left(R_{+}, \rho\right)\right] .
$$

Under the action of a similarity transformation with operator $H$, shift operator $W_{R} \in\left\lfloor L_{2}(R)\right\rfloor$ is transformed into a matrix multiplication operator $\mathbf{V}_{R_{+}}$in $L_{2}^{2}\left(R_{+}, \rho(t)\right)$ :

$$
H W_{R} H^{-1}=\mathbf{V}_{R_{+}}, \quad \mathbf{V}_{R_{+}}=\left[\begin{array}{cc}
1 & 0 \\
0 & -1
\end{array}\right]
$$

We also indicate that the operator of multiplication by a scalar function $a_{R}(t) I_{R}$ becomes:

$$
\begin{gathered}
H a_{R}(t) I_{R} H^{-1}= \\
\frac{1}{2}\left[\begin{array}{ll}
a\left[_{R}(\sqrt{t})+a_{R}(-\sqrt{t})\right] & {\left[a_{R}(\sqrt{t})-a_{R}(-\sqrt{t})\right]} \\
a\left[_{R}(\sqrt{t})-a_{R}(-\sqrt{t})\right] & {\left[a_{R}(\sqrt{t})+a_{R}(-\sqrt{t})\right]}
\end{array}\right] .
\end{gathered}
$$

These formulas can be verified by direct calculations. The functional operator with shift, as follows from (1) and (2), becomes the operator of multiplication by a matrix function in $L_{2}^{2}\left(R_{+}, \rho(t)\right)$ :

$$
H\left[a_{R}(t) I_{R}+b_{R}(t) W_{R}\right] H^{-1}=\frac{1}{2}\left[\begin{array}{ll}
g_{11}(t) & g_{12}(t) \\
g_{21}(t) & g_{22}(t)
\end{array}\right],
$$

where

$$
\begin{aligned}
& g_{11}(t)=\left[a_{R}(\sqrt{t})+b_{R}(\sqrt{t})\right]+\left[\mathrm{b}_{R}(-\sqrt{t})+a_{R}(-\sqrt{t})\right], \\
& g_{12}(t)=\left[a_{R}(\sqrt{t})-b_{R}(\sqrt{t})\right]+\left[\mathrm{b}_{R}(-\sqrt{t})-a_{R}(-\sqrt{t})\right], \\
& g_{21}(t)=\left[a_{R}(\sqrt{t})+b_{R}(\sqrt{t})\right]-\left[\mathrm{b}_{R}(-\sqrt{t})+a_{R}(-\sqrt{t})\right], \\
& g_{22}(t)=\left[a_{R}(\sqrt{t})-b_{R}(\sqrt{t})\right]-\left[\mathrm{b}_{R}(-\sqrt{t})-a_{R}(-\sqrt{t})\right] .
\end{aligned}
$$


The formula constructed is very important for what follows, since it connects function operators with reflection with matrix functions of second order. Due to (3), the factorization factors in the multiplicative representation of the matrix function will be represented as the corresponding functional operators with reflection, and the factor with the partial indices of the matrix will be an operator of a special form with shift, which is one-to-one determined by these partial indices.

\section{Factorization of Functional \\ Operators with Reflection}

We review known definitions from $[11,12]$. Let $L_{2}^{+}(R, \rho)=P_{R}^{+} L_{2}(R, \rho), \quad L_{2}^{-}(R, \rho)=P_{R}^{-} L_{2}(R, \rho)$, where $P_{R}^{ \pm}$are the projections associated with the Cauchy singular integral operator along $R$ :

$P_{R}^{ \pm}=\frac{1}{2}\left(I_{R} \pm S_{R}\right), \quad\left(S_{R} \varphi\right)(t)=\frac{1}{\pi i} \int_{R} \frac{\varphi(\tau)}{\tau-t} d \tau$.

Singular integral operator $S_{R}$ acts boundedly in the space with weight $L_{2}(R, \rho), \rho(t)=t^{-1 / 4}$. Let us introduce similar notation for operators acting in the space of vector functions $L_{2}^{2}(R, \rho)$ :

$$
\mathbf{I}_{R}, \mathbf{S}_{R}, \mathbf{P}_{R}^{+}=\frac{1}{2}\left(\mathbf{I}_{R}+\mathbf{S}_{R}\right), \mathbf{P}_{R}^{-}=\frac{1}{2}\left(\mathbf{I}_{R}-\mathbf{S}_{R}\right) .
$$

We also introduce vector spaces:

$L_{2}^{2+}(R, \rho)=\mathbf{P}_{R}^{+} L_{2}^{2}(R, \rho), L_{2}^{2-}(R, \rho)=\mathbf{P}_{R}^{-} L_{2}^{2}(R, \rho)$.

By $L_{\infty}(\Gamma)$ we denote the set of all measurable essentially bounded functions on the contour $\Gamma$, by $L_{\infty}^{2}(\Gamma)$ we denote the set of all matrix functions with elements from $L_{\infty}(\Gamma)$.

We give a definition of factorization of a matrix function.

\section{Definition 1}

Factorization of non-degenerate matrix function $\mathbf{G}(t) \in L_{\infty}^{2}(\mathrm{R})$ in the space $L_{2}^{2}(R, \rho), \rho(t)=t^{-1 / 4}$ is expressed as

$$
\mathbf{G}(t)=\boldsymbol{\Psi}^{+}(t) \boldsymbol{\Lambda}_{R}(t) \boldsymbol{\Psi}^{-}(t),
$$

were matrix functions $\boldsymbol{\Psi}^{ \pm}(t)$ possess the properties:

$$
\begin{aligned}
& \frac{\boldsymbol{\Psi}^{+}(t)}{t+i} \in L_{2}^{2+}(R, \rho), \frac{\left[\boldsymbol{\Psi}^{-}\right]^{-1}(t)}{t-i} \in L_{2}^{2-}(R, \rho), \\
& \frac{\left[\boldsymbol{\Psi}^{+}\right]^{-1}(t)}{t+i} \in L_{2}^{2+}\left(R, \rho^{-1}\right), \frac{\boldsymbol{\Psi}^{-}(t)}{t-i} \in L_{2}^{2-}\left(R, \rho^{-1}\right),
\end{aligned}
$$

and were

$$
\boldsymbol{\Lambda}_{R}(t)=\operatorname{diag}\left\lfloor\left(\frac{1-t}{1+t}\right)^{\kappa_{1}},\left(\frac{1-t}{1+t}\right)^{\kappa_{2}}\right\rfloor,
$$

numbers $\kappa_{1}, \kappa_{2}$ are integers and $\kappa_{1} \geq \kappa_{2}$.

The numbers $\kappa_{1}, \kappa_{2}$ are called partial indices of the matrix function $\mathbf{G}(t)$ and their sum is called the total index. It is known [11, 12] that the partial indices are invariants of factorization and they do not depend on a particular type of representation (4). That is, they are uniquely defined by $\mathbf{G}(t)$.

Let us give a definition of factorization of a functional operator with shift-reflection based on operator identities and the definition of factorization of matrix functions. To do this, we use the operator $H \in\left[L_{2}(R), L_{2}^{2}\left(R_{+}, \rho\right)\right]$ and its property (3). Let functions $a_{R}(t), b_{R}(t), c_{R}(t)$, $d_{R}(t)$ belong to $L_{\infty}(\mathrm{R})$.

\section{Definition 2}

Factorization of the invertible functional operator

$$
A=a_{R}(t) I_{R}+b_{R}(t) W_{R}, \quad A \in\left[L_{2}(\mathrm{R})\right]
$$

with the invertible continuation operator

$$
K=c_{R}(t) I_{R}+d_{R}(t) W_{R},
$$

will be called its representation in the form

$$
A=
$$

$\left[A^{+}(t) I_{R}+B^{+}(t) W_{R}\right] \Omega(t)\left[A^{-}(t) I_{R}+B^{-}(t) W_{R}\right]$

under the continuation condition

$$
K=
$$

$\left[C^{+}(t) I_{R}+D^{+}(t) W_{R}\right] \Pi(t)\left[C^{-}(t) I_{R}+D^{-}(t) W_{R}\right]$,

where

$$
\begin{gathered}
\Omega(t)=H^{-1}\left[\boldsymbol{\Lambda}_{R}(t)\right] H, \\
\Pi(t)=H^{-1}\left[\boldsymbol{\Lambda}_{R}(-t) \boldsymbol{\Lambda}_{R}^{-1}(t)\right] H \Omega(t) .
\end{gathered}
$$

Moreover, the matrix functions

$$
\boldsymbol{\Psi}^{ \pm}(t)=
$$

$$
\mathbf{J}_{R_{-}}\left\lfloor\mathbf{A}_{R_{+}}^{ \pm}+\mathbf{B}_{R_{+}}^{ \pm} \mathbf{V}_{R_{+}}\right\rfloor+\mathbf{W}_{R} \mathbf{J}_{R_{-}}\left\lfloor\mathbf{C}_{R_{+}}^{ \pm}+\mathbf{D}_{R_{+}}^{ \pm} \mathbf{V}_{R_{+}}\right\rfloor,
$$

where

$$
\begin{aligned}
& \left\lfloor\mathbf{A}_{R_{+}}^{ \pm}+\mathbf{B}_{R_{+}}^{ \pm} \mathbf{V}_{R_{+}}\right\rfloor=H\left[A^{ \pm}(t) I_{R}+B^{ \pm}(t) W_{R}\right] H^{-1},(7) \\
& \left\lfloor\mathbf{C}_{R_{+}}^{ \pm}+\mathbf{D}_{R_{+}}^{ \pm} \mathbf{V}_{R_{+}}\right\rfloor=H\left[C^{ \pm}(t) I_{R}+D^{ \pm}(t) W_{R}\right] H^{-1}
\end{aligned}
$$


should not degenerate and should have properties (E):

$$
\frac{\left(\mathbf{J}_{R_{-}}\left[\mathbf{A}_{R_{+}}^{ \pm}+\mathbf{B}_{R_{+}}^{ \pm} \mathbf{V}_{R_{+}}\right]+\mathbf{W}_{R} \mathbf{J}_{R_{-}}\left[\mathbf{C}_{R_{+}}^{ \pm}+\mathbf{D}_{R_{+}}^{ \pm} \mathbf{V}_{R_{+}}\right]\right)^{ \pm 1}}{t \pm i}
$$

belongs to $L_{2}^{2 \pm}(R, \rho)$ and

$$
\frac{\left(\mathbf{J}_{R_{-}}\left[\mathbf{A}_{R_{+}}^{ \pm}+\mathbf{B}_{R_{+}}^{ \pm} \mathbf{V}_{R_{+}}\right]+\mathbf{W}_{R} \mathbf{J}_{R_{-}}\left[\mathbf{C}_{R_{+}}^{ \pm}+\mathbf{D}_{R_{+}}^{ \pm} \mathbf{V}_{R_{+}}\right]\right)^{\mp 1}}{t \pm i}
$$

belongs to $L_{2}^{2 \pm}(R, \rho)$.

\section{Relationship between the}

factorization of functional operators with reflection and the factorization of the corresponding matrix functions

Let an invertible functional operator $A=a_{R}(t) I_{R}+b_{R}(t) W_{R}, c_{R}(t), d_{R}(t) \in L_{\infty}(\mathrm{R})$

Admit factorization in $L_{2}(\mathrm{R})$ with a continuation operator

$K=c_{R}(t) I_{R}+d_{R}(t) W_{R}, \quad c_{R}(t), d_{R}(t) \in L_{\infty}(\mathrm{R})$.

We introduce a matrix function $\mathbf{G}_{R_{+}}(t)$ defined on the positive semi-axis $R_{+}$that equals $H A H^{-1}$ and a matrix function $\mathbf{K}(-t)$ defined on $R_{+}$that equals $H K H^{-1}$. From these operators $\mathbf{G}_{R_{+}}(t)=H A H^{-1}$ and $\mathbf{K}(-t)=H K H^{-1}$, we compose a matrix function, defined on the whole real axis $\mathrm{R}$ :

$$
\mathbf{G}_{R}^{\mathbf{K}}(t)=\mathbf{J}_{R_{-}} \mathbf{G}_{R_{+}}(t)+\mathbf{W}_{R} \mathbf{J}_{R_{-}} \mathbf{K}(-t) .
$$

Note that $\mathbf{K}(t)$ is a vector function defined on the negative semi axis $\mathrm{R}_{-}$.

And now, we will prove that this matrix function $\mathbf{G}_{R}^{\mathbf{K}}(t)$ admits factorization

$$
\mathbf{G}_{R}^{\mathbf{K}}(t)=\boldsymbol{\Psi}_{R}^{+}(t) \boldsymbol{\Lambda}_{R}(t) \boldsymbol{\Psi}_{R}^{-}(t)
$$

in space $L_{2}^{2}(R, \rho), \rho(t)=t^{-1 / 4}$.

We carry out transformations of $\mathbf{G}_{R}^{\mathbf{K}}(t)=$ $\mathbf{J}_{R_{-}} H H^{-1} \mathbf{G}_{R_{+}}(t) H H^{-1}+\mathbf{W}_{R} \mathbf{J}_{R_{-}} H H^{-1} \mathbf{K}(-t) H H^{-1}=$ $\mathbf{J}_{R_{-}} H\left[a_{R} I_{R}+b_{R} \mathrm{~W}_{R}\right] H^{-1}+\mathbf{W}_{R} \mathbf{J}_{R_{-}} H\left[\mathrm{c}_{R} I_{R}+d_{R} \mathrm{~W}_{R}\right] H^{-1}$, then use representations (5), (6) and continue calculations of $\mathbf{G}_{R}^{\mathbf{K}}(t)$

$$
\begin{aligned}
& \mathbf{J}_{R_{-}}\left(H\left[A^{+}(t) I_{R}+B^{+}(t) \mathrm{W}_{R}\right] H^{-1} H \Omega(t) H^{-1} \times\right. \\
& \left.H\left[A^{-}(t) I_{R}+B^{-}(t) \mathrm{W}_{R}\right] H^{-1}\right)+
\end{aligned}
$$

$\mathbf{W}_{R} \mathbf{J}_{R_{-}}\left(H\left[C^{+}(t) I_{R}+D^{+}(t) \mathrm{W}_{R}\right] H^{-1} H[\Pi(t)] H^{-1} \times\right.$ $\left.H\left[C^{-}(t) I_{R}+D^{-}(t) \mathrm{W}_{R}\right] H^{-1}\right)=$

using representation (7), we continue the calculations of $\mathbf{G}_{R}^{\mathbf{K}}(t)$

$\mathbf{J}_{R_{-}}\left(\left[\mathbf{A}_{R_{+}}^{+}+\mathbf{B}_{R_{+}}^{+} \mathbf{V}_{R_{+}}\right]\left[C_{R_{+}} \boldsymbol{\Lambda}_{R}(t)\right]\left[\mathbf{A}_{R_{+}}^{-}+\mathbf{B}_{R_{+}}^{-} \mathbf{V}_{R_{+}}\right]\right)+\mathbf{W}_{R^{\prime}} \mathbf{J}_{R_{-}}$ $\left(\left[\mathbf{C}_{R_{+}^{+}}^{+}+\mathbf{D}_{R_{+}}^{+} \mathbf{V}_{R_{+}}\right]\left[C_{R_{+}} \boldsymbol{\Lambda}_{R}(-t)\right]\left[\mathbf{C}_{R_{+}}^{-}+\mathbf{D}_{R_{+}}^{-} \mathbf{V}_{R_{+}}\right]\right)$.

Finally we get $\mathbf{G}_{R}^{\mathbf{K}}(t)=$ $\mathbf{J}_{R_{-}}\left(\left[\mathbf{A}_{R_{+}}^{+}+\mathbf{B}_{R_{+}}^{+} \mathbf{V}_{R_{+}}\right]+\mathbf{W}_{R} \mathbf{J}_{R_{-}}\left[\mathbf{C}_{R_{+}}^{+}+\mathbf{D}_{R_{+}}^{+} \mathbf{V}_{R_{+}}\right]\right) \boldsymbol{\Lambda}_{R}(t) \times$ $\mathbf{J}_{R_{-}}\left(\left[\mathbf{A}_{R_{+}}^{-}+\mathbf{B}_{R_{+}}^{-} \mathbf{V}_{R_{+}}\right]+\mathbf{W}_{R} \mathbf{J}_{R_{-}}\left[\mathbf{C}_{R_{+}}^{-}+\mathbf{D}_{R_{+}}^{-} \mathbf{V}_{R_{+}}\right]\right)$.

From the definition of factorization of the operator $A$ with continuation operator $K$, it follows that the factors

$\boldsymbol{\Psi}_{R}^{+}(t)=$

$\mathbf{J}_{R_{-}}\left[\mathbf{A}_{R_{+}}^{+}(t)+\mathbf{B}_{R_{+}}^{+}(t) \mathbf{V}_{R_{+}}\right]+\mathbf{W}_{R} \mathbf{J}_{R_{-}}\left[\mathbf{C}_{R_{+}}^{+}(t)+\mathbf{D}_{R_{+}}^{+}(t) \mathbf{V}_{R_{+}}\right]$,

$\boldsymbol{\Psi}_{R}^{-}(t)=$

$\mathbf{J}_{R_{-}}\left[\mathbf{A}_{R_{+}}^{-}(t)+\mathbf{B}_{R_{+}}^{-}(t) \mathbf{V}_{R_{+}}\right]+\mathbf{W}_{R} \mathbf{J}_{R_{-}}\left[\mathbf{C}_{R_{+}}^{-}(t)+\mathbf{D}_{R_{+}}^{-}(t) \mathbf{V}_{R_{+}}\right]$

possess property (E). Hence, it follows that the matrix function $\mathbf{G}_{R}^{\mathbf{K}}(t)$ admits factorization.

Note that $\boldsymbol{\Psi}_{R}^{ \pm}(t), t \in R$, are boundary values of analytic non-singular matrix functions in the upper and lower half-plane, respectively.

We shall now prove the converse statement. Assuming the admissibility of factorization of some matrix function $\tilde{\mathbf{G}}_{R}$ in $L_{2}^{2}(R, \rho)$ :

$$
\tilde{\mathbf{G}}_{R}(t)=\tilde{\boldsymbol{\Psi}}_{R}^{+}(t) \boldsymbol{\Lambda}_{R}(t) \tilde{\boldsymbol{\Psi}}_{R}^{-}(t) .
$$

Let us build functional operators $A$ and $K$ which are related to $\tilde{\mathbf{G}}_{R}$ in the following way:

$a_{R}(t) I_{R}+b_{R}(t) W_{R}=H^{-1}\left[C_{R_{+}} \tilde{\mathbf{G}}_{R}(t)\right] H, A \in L_{2}(R)$, $c_{R}(t) I_{R}+d_{R}(t) W_{R}=H^{-1}\left[C_{R_{+}} \tilde{\mathbf{G}}_{R}(-t)\right] H, K \in L_{2}(R)$.

Now, we will prove the admissibility of factorization of the functional operator $A=a_{R}(t) I_{R}+b_{R}(t) W_{R} \quad$ in $L_{2}(\mathrm{R}) \quad$ with the continuation $K=c_{R}(t) I_{R}+d_{R}(t) W_{R}, K \in L_{2}(R)$. If we denote

$\left[C_{R_{+}} \tilde{\mathbf{G}}_{R}(t)\right]=\tilde{\mathbf{G}}_{R_{+}}(t)$ and $\left[C_{R_{-}} \tilde{\mathbf{G}}_{R}(t)\right]=\tilde{\mathbf{K}}(t)$, we get 


$$
\tilde{\mathbf{G}}_{R}(t)=\mathbf{J}_{R_{-}} \tilde{\mathbf{G}}_{R_{+}}(t)+\mathbf{W}_{R} \mathbf{J}_{R_{-}} \tilde{\mathbf{K}}(-t)
$$

and

$$
A=H^{-1} \tilde{\mathbf{G}}_{R_{+}}(t) H, \quad K=H^{-1} \tilde{\mathbf{K}}(-t) H .
$$

Factorization representation of the matrix $\tilde{\mathbf{G}}_{R}(t)$ can be written in an equivalent form:

$\tilde{\mathbf{G}}_{R_{+}}(t)=\tilde{\boldsymbol{\Psi}}_{R}^{+}(t) \boldsymbol{\Lambda}_{R}(t) \tilde{\boldsymbol{\Psi}}_{R}^{-}(t), \quad t \in R_{+} ;$

$\tilde{\mathbf{K}}(-t)=\tilde{\boldsymbol{\Psi}}_{R}^{+}(-t) \boldsymbol{\Lambda}_{R}(-t) \tilde{\mathbf{\Psi}}_{R}^{-}(-t), \quad t \in R_{+}$.

Applying operator $H^{-1}$ on the left side, and operator $H$ on the right side to the factorization representation, we obtain:

$$
\begin{gathered}
H^{-1} \tilde{\mathbf{G}}_{R_{+}}(t) H= \\
H^{-1} \tilde{\mathbf{\Psi}}_{R}^{+}(t) H\left[H^{-1} \boldsymbol{\Lambda}_{R}(t) H\right] H^{-1} \tilde{\mathbf{\Psi}}_{R}^{-}(t) H, t \in R_{+} ; \\
H^{-1} \tilde{\mathbf{K}}(-t) H= \\
H^{-1} \tilde{\boldsymbol{\Psi}}_{R}^{+}(-t) H\left[H^{-1} \boldsymbol{\Lambda}_{R}(-t) H\right] \tilde{\boldsymbol{\Psi}}_{R}^{-}(-t) H^{-1}, t \in R_{+} .
\end{gathered}
$$

Proceeding to the functional operators with reflection, we have:

$$
\begin{gathered}
a_{R}(t) I_{R}+b_{R}(t) W_{R}= \\
{\left[A^{+}(t) I_{R}+B^{+}(t) W_{R}\right] \Omega(t)\left[A^{-}(t) I_{R}+B^{-}(t) W_{R}\right],} \\
c_{R}(t) I_{R}+d_{R}(t) W_{R}= \\
{\left[C^{+}(t) I_{R}+D^{+}(t) W_{R}\right] \Pi(t)\left[C^{-}(t) I_{R}+D^{-}(t) W_{R}\right],}
\end{gathered}
$$

where

$$
\begin{aligned}
& {\left[H^{-1} \tilde{\boldsymbol{\Psi}}_{R}^{-}(t) H\right]=\left[A^{+}(t) I_{R}+B^{+}(t) W_{R}\right],} \\
& {\left[H^{-1} \tilde{\boldsymbol{\Psi}}_{R}^{-}(t) H\right]=\left[A^{-}(t) I_{R}+B^{-}(t) W_{R}\right],} \\
& {\left[H^{-1} \tilde{\boldsymbol{\Psi}}_{R}^{+}(-t) H\right]=\left[C^{+}(t) I_{R}+D^{+}(t) W_{R}\right],} \\
& {\left[H^{-1} \tilde{\boldsymbol{\Psi}}_{R}^{-}(-t) H\right]=\left[C^{-}(t) I_{R}+D^{-}(t) W_{R}\right] .}
\end{aligned}
$$

We remind that $\Omega(t)=H^{-1}\left[C_{R_{+}} \Lambda_{R}(t)\right] H$ and $\Pi(t)=H^{-1}\left[\boldsymbol{\Lambda}_{R}(-t) \boldsymbol{\Lambda}_{R}^{-1}(t)\right] H \Omega(t)$.

It remains to make sure that in the representation of the matrix function $\tilde{\mathbf{G}}_{R}(t)$, factors $\tilde{\mathbf{\Psi}}^{ \pm}(t)$ have properties (E). Functions $\tilde{\mathbf{\Psi}}^{ \pm}(t)$ can be represented as

$$
\begin{aligned}
& \mathbf{J}_{R_{-}} H H^{-1}\left[C_{R_{+}} \tilde{\mathbf{\Psi}}_{R}^{ \pm}(t)\right] H H^{-1}+ \\
& \mathbf{W}_{R} \mathbf{J}_{R_{-}} H H^{-1}\left[C_{R_{+}} \tilde{\mathbf{\Psi}}_{R}^{ \pm}(-t)\right] H H^{-1}=
\end{aligned}
$$

$$
\begin{aligned}
& \mathbf{J}_{R_{-}} H\left[A^{ \pm}(t) I_{R}+B^{ \pm}(t) W_{R}\right] H^{-1}+ \\
& \mathbf{W}_{R} \mathbf{J}_{R_{-}} H\left[C^{ \pm}(t) I_{R}+D^{ \pm}(t) W_{R}\right] H^{-1}
\end{aligned}
$$

It follows that the required properties (E) are fulfilled. So we have proven the theorem.

\section{Theorem}

An invertible functional operator with reflection

$$
A=a_{R}(t) I_{R}+b_{R}(t) W_{R}, c_{R}(t), d_{R}(t) \in L_{\infty}(\mathrm{R})
$$

and with an invertible continuation operator

$$
K=c_{R}(t) I_{R}+d_{R}(t) W_{R}, \quad c_{R}(t), d_{R}(t) \in L_{\infty}(\mathrm{R})
$$

admits factorization in $L_{2}(\mathrm{R})$ if and only if the corresponding non-singular matrix function $\mathbf{G}_{R}(t)$ admits factorization in $L_{2}^{2}(R, \rho)$, where the operators are related by the formulas:

$$
\begin{gathered}
\mathbf{G}_{R}(t)=\mathbf{J}_{R_{-}}\left[H^{-1} A H\right]+\mathbf{W}_{R} \mathbf{J}_{R_{-}}\left[H^{-1} K H\right], \\
A=H^{-1}\left[C_{R_{+}} \mathbf{G}_{R}(t)\right] H, \quad K=H^{-1}\left[\mathbf{G}_{R}(-t)\right] H .
\end{gathered}
$$

\section{Corollary}

Partial indices $\kappa_{1}, \kappa_{2}$ of the invertible functional operator with reflection $A=a_{R}(t) I_{R}+b_{R}(t) W_{R}$ and continuation $K=c_{R}(t) I_{R}+d_{R}(t) W_{R}$ that admits factorization in $L_{2}(\mathrm{R})$ are uniquely determined. They do not depend on a particular type of factorization representation of operators $A, K$ and coincide with the partial indices of the corresponding matrix function $\mathbf{G}_{R}(t)$.

\section{Discussion}

An effective solution of Riemann boundary value problem has not yet been found and the problem of factorization of matrix functions is closely connected with effective solution of matrix Riemann problem. Therefore, advances in these areas are valuable. Recently, there have been works devoted to the factorization of special cases of matrix functions, estimates for partial indices and approximate methods. Our research has a different focus. We wanted to apply another mathematical apparatus to the study of factorization problems: functional 
operators with Carleman shifts and operator identities. In [12], for these purposes, functional operators with Carleman orientation preserving rotations were used. In this paper, we used functional operators and operator identities with reversing reflection on the real axis. Note a significant difference from [12]: when applying the operator identity to a functional operator with reflection, we obtain the corresponding matrix function, but on a part of the contour, the semi axis. The question arises of extending the matrix to the entire axis. This will be a topic for further study of factorization of functional operators.

\section{Conclusion}

This paper presents the concept of factorization of functional operators with shift-reflection on the real axis. The main method of investigation is operator identities, which are similarity transformation with reciprocal operators $H, H^{-1}$ constructed by the authors: $H A H^{-1}=\mathbf{G}_{R_{+}}$. Based on this relation, the theorem on the equivalence of factorization of matrix functions and factorization of functional operators with shift-reflection was proven.

Singular integral equations with Carleman linear fractional shift and their corresponding scalar Riemann boundary value problems with shift are equivalently transformed with the action of operator identities in characteristic vector singular integral equations without shift and in their corresponding matrix Riemann boundary value problems without shift. It is known that factorization problems for matrix functions are closely related to matrix Riemann boundary value problems. In [15, p.24], a method scheme is proposed that shows how, using the known factorization of a matrix, a solution to the matrix Riemann problem function is obtained. Through the application of operator identities, we identified a range of equivalent problems that makes it possible to transfer results obtained in one problem to other problems.

\section{References:}

[1] Rogosin S., Mishuris G., Constructive Methods for Factorization of MatrixFunctions, IMA Journal of Applied Mathematics, Vol.81, No.2, 2016, pp. 365391.

[2] Harutyunyan K., V., Kamalyan A., G., Spitkovsky I., M., On the Partial Indices of Triagular Matrix Function with Prescribed Indices of Their Diagonal Entries, Operator Theory in Different Settings and Related Applications, 2017, pp. 251-285.

[3] Primachuk L., Rogosin S., Factorization of Triangular Matrix - Function, Lobachevskii Mathematica Journal, Vol.39, 2018, pp. 129-137

[4] Mishuris G., Rogosin S., Regular Approximate Factorization of a Class of of Matrix-Function with an Nnstable Set of Partial Indices, Proceedings of the Royal Society a Mathematical, Fhysical and Engineering Scieces, Vol.474, 2018, pp. 1-18.

[5] Demjanovich Yu., K., Evdokimova T., O., Ivancova O., N., Lebedinskii D., M., Ponomareva A., Y., On Wavelet Decomposition of the Singular Splines, International Journal of Circuits, Systems and Signal Processing, Vol.14, 2020, pp. 571-579.

[6] Gusic D., Approximate Formulas for Zeta Functions of Selberg's Type in Quotients of SL4, International Journal of Circuits, Systems and Signal Processing, Vol.14, 2020, pp. 21-27.

[7 Karelin A. A., On a Relation between Singular Integral Operators with a Carleman Linear - Fractional Shift and Matrix Characteristic Operators without Swift, Boletín de Sociedad Matemática Mexicana, Vol.7, No.3, 2001, pp. 235-246.

[8] Karelin A. A., On the Operator Equality and Some of Its Application, Proceedings of A. Razmadze Mathematical Institute, Vol.128, 2002, pp. 105-116.

[9] Karelin A., Applications of Operator Equalities to Singular Integral Operators and Riemann Boundary Value Problems, Mathematische Nachrichten, Vol.280, No.9-10, 2007, pp. 1108-1117.

[10] Karelin A. A., Singular Integral Operators with Coefficients of a Special Structure Related to Operator Equalities, Complex Analysis and Operator Theory, Vol.2, No.4, 2008, pp. 549-567.

[11] Karelin A. A., Perez-Lechuga G, Tarasenko A.A., Riemann Problem and Singular Integral Equations with Coefficients Generated by Piecewise Constant Functions, Differential Equations, Vol.44, No. 9, 2008, pp. 1182-1192. 
[12] Karelin A., Tarasenko A., Factorization of Functional Operators with Involutive Rotation on the Unit Circle, Applied Mathematics, Scientific Research Publishing, Vol.11, 2020, pp. 1132-1138.

[13] Spitkovski I. M., Tashbaev, A. M., Factorization of Certain Piecewise Constant Matrix Functions and Its Aplication, Mathematische Nachrichten, Vol.151, No.1, 1997, pp. 241-261.

[14] Litvinchuk G., S., Spitkovskii I.. M., Factorization of Measurable Matrix Functions, Operator Theory: Advances and Application, Vol.25, Birkhauser Verlag, Basel, Boston, 1987.

[15 ] Litvinchuk G., S., Solvability theory of boundary value problems and singular integral equations with shift, KluwerAcad. Publ., Dordrecht, Boston, London, 2000.

Oleksandr Karelin and Anna Taresenko contributed equally to the creation of this article.

\section{Creative Commons Attribution License 4.0 (Attribution 4.0 International, CC BY 4.0)}

This article is published under the terms of the Creative Commons Attribution License 4.0

https://creativecommons.org/licenses/by/4.0/deed.en_US 